\title{
Revisit percutaneous dissolution of uric acid stones: A case report with review
}

\begin{abstract}
Uric acid stones are second most common cause of kidney stones after calcium oxalate and calcium phosphate stones. Its prevalence varies based on the age, climate, ethnicity, and geographic location. Here we are presenting a case report where a 55 -years old male with a history of prostate cancer and MGUS presented with acute renal failure due to bilateral ureteral obstruction with uric acid stones. The computer tomography (CT) scan of abdomen and pelvis showed two obstructive stones measuring $28 \mathrm{~mm}$ and $30 \mathrm{~mm}$ in the left proximal ureter and one obstructive stone measuring $30 \mathrm{~mm}$ in the right mid ureter. The first line conservative management with hydration failed as patient becomes significant volume overload. He was successfully treated with percutaneous chemolysis using a $3 \mathrm{amps}$ of bicarbonate in $20 \mathrm{cc}$ solution every six hours and potassium citrate (20meq) $2160 \mathrm{mg}$ three times in a day. By hospital day five majority of his stones resolved and repeat CT scan showed only punctate nonobstructing calcifications. The usual treatment of uric acid nephrolithiasis consists of hydration to produce at least $2 \mathrm{~L}$ of urine volume daily, low animal protein diet, and urine alkalization. Although localized alkalization via retrograde or percutaneous is now an uncommon practice due to longer hospital stays and cost effectiveness. However, physicians should be aware of this effective alternative especially in situations with complete bilateral ureteral obstruction, severe metabolic disorders, acute renal failure, or fluid overload.
\end{abstract}

Keywords: Uric acid stones, nephrolithiasis, bilateral ureteral obstruction, percutaneous chemolysis
Volume 6 Issue 5 - 2018

\author{
Amarpreet Sandhu,' Karen Servilla, ${ }^{2}$ Curtis \\ Kapsner, ${ }^{2}$ Anthony Smith ${ }^{3}$ \\ 'Department of Kidney Transplant, California Pacific Medical \\ Center, USA \\ ${ }^{2}$ Department of Nephrology and Urology, University of New \\ Mexico and Veterans Affairs Hospital, Mexico
}

\author{
Correspondence: Dr. Amarpreet Sandhu, Transplant \\ Nephrologist, Department of Kidney Transplant, California \\ Pacific Medical Center, San Francisco, USA, \\ Email sandhudoctor@gmail.com
}

Received: June 27, 2018 | Published: October 02, 2018

\section{Introduction}

Uric acid stones account for $5-10 \%$ of all kidney stones in the United States. Its prevalence can vary by age, climate, ethnicity, and geographic location making Israel one of the countries with uric acid stone incidences of $40 \%$. Acidic $\mathrm{pH}(<5.5)$ is a major risk factor in uric acid stone formation. Prevention is a key to manage uric acid nephrolithiasis. The conservative treatment of uric acid stones includes increased fluid intake to alkalization of urine either systemically or locally. Surgery is the last resort. Localized dissolution of uric acid stones is an effective non-surgical approach that was a common practice in the past. With the effectiveness of systemic alkalization and longer hospital stays for localized dissolution of uric acid stones, it has become less favorable overtime. However, it should be considered as first line treatment in situation with complete bilateral ureteral obstruction, severe metabolic disorders, severe renal failure, or fluid overload, as seen in our presented case report. With a coordinated and timely care by specialists, the hospital stay can be shortened making this modality much more cost effective.

\section{Prevalence}

The prevalence of uric Acid stones accounts for 5\%-10\% of all kidney stones in the United States. In a study by Mandel ${ }^{1}$ at Veterans Administration hospitals revealed that $9.7 \%$ analyzed stones were pure uric acid stones whereas, $12 \%$ of the stones contained some uric acid components. ${ }^{1}$ The prevalence of uric acid stones can vary by age, climate, ethnicity, and geographic location. The history of diabetes and obesity increase the risk of stone formation. ${ }^{2}$ Gentle et al. ${ }^{3}$ reported uric acid stone prevalence of $11 \%$ in a geriatric population.
Higher uric acids stone incidences are reported in the factory employees working in the higher temperature environments that is likely the contributor of highest levels of uric acids stones reported in the Israel. ${ }^{4}$ Approximately $50 \%$ of patients with nephrolithiasis in the Hmong population of the United States are reported to have uric acid stones. Geographically, only $4 \%$ of uric acid nephrolithiasis have been reported in the southern states of the United States; whereas, incidence rates are $17 \%$ in Chicago..$^{5}$ Outside of the United States, the incidence of uric acid stones varies such as, $4 \%$ in Sweden, $15 \%$ in Japan, $20 \%-25 \%$ in Germany, and $40 \%$ in Israel. ${ }^{6}$ These variations indicate that genes, diet, and environmental factors play major role in formation of uric acid stones.

\section{Risk factors}

Two main risk factors for uric acid stones formation are low urinary $\mathrm{pH}$ and hyperuricosuria. Of these two major risk factors, acidic (low, <5.5) urinary $\mathrm{pH}$ is most common and it is usually caused by tubular disorders, low urinary volume, chronic diarrhea, or severe dehydration. Hyperuricuria ( $>800 \mathrm{mg}$ of urinary uric acid/24hrs) can be associated with hyperuricemia, such as in primary gout or myeloproliferative disorder, or may manifest as an isolated cause due to diet or uricosuric drugs. ${ }^{7}$ The monogenic metabolic conditions such as Lesch-Nyhan also predisposes to uric acid nephrolithiasis. A gene ZNF365 located on chromosome 10q21-q22 linked to uric acid calculus formation is detected but its purpose is not well defined yet. ${ }^{8}$

Recent analysis from multiple retrospective studies by Daudon et al. ${ }^{9}$ has concluded that within frequent stones formers, uric acids stones are most common in patients who have diabetes, obesity, or metabolic syndrome. ${ }^{9}$ 


\section{Case report}

55-yrs-old-male with history of prostate cancer Gleason 3 plus 4 status post radical prostectomy 3 weeks ago presented with 2-days of nausea, vomiting, weakness, decreased urine output, and pre-syncopal episode. His past medical history included MGUS, hypertension, depression, nocardial osteomyelitis, pyelonephritis, and megaloblastic anemia. His initial vital signs were abnormal with respiratory rate of $32 \mathrm{rpm}$ and systolic blood pressures of $80 \mathrm{mmHg}$. En-route from outside hospital he received 9 liters of IVFs for hypotension. On presentation to emergency department, he was fluid overload and got intubated for respiratory distress. His laboratory work up was abnormal with $\mathrm{pH}$ of $7.15, \mathrm{PCO}_{2} 26 \mathrm{mmHg}, \mathrm{Hgb} 6.2 \mathrm{~g} / \mathrm{dl}$, glucose $36 \mathrm{mg} / \mathrm{dl}$, Potassium $6 \mathrm{mmol} / \mathrm{L}, \mathrm{CO}_{2} 9 \mathrm{mmol} / \mathrm{L}$, phosphorus $9.9 \mathrm{mg} / \mathrm{dl}$, albumin $3.1 \mathrm{~g} / \mathrm{dl}$, amylase $181 \mathrm{U} / \mathrm{L}$, lipase $364 \mathrm{U} / \mathrm{L}, \mathrm{BUN} 98 \mathrm{mg} / \mathrm{dl}$, and Creatinine $9.8 \mathrm{mg} / \mathrm{dl}$.

Computer tomography (CT) scan of abdomen and pelvis showed two obstructive stones measuring $28 \mathrm{~mm}$ and $30 \mathrm{~mm}$ in the left proximal ureter and one obstructive stone measuring $30 \mathrm{~mm}$ in the right mid ureter. Emergently right femoral vascular catheter was placed and received 2 hours of dialysis for volume overload, electrolyte and acid base disorder. Few hours later bilateral nephrostomy tubes were placed by interventional radiologist and drained very little urine volume. His urine was examined under microscope and showed uric acid crystals, few granular casts, and many normo-morphic red blood cells.

Overnight he continued to drain from both nephrostomies but urine output was not significant. He was started on local irrigation with a solution of 3 amps of bicarbonate in $20 \mathrm{cc}$, every six hours. He also received potassium citrate (20meq) $2160 \mathrm{mg}$ three times a day, initially via nasogastric tube and later by mouth. The following day, the patient's urine output increased, he was extubated, and his dialysis catheter was discontinued. His BUN and creatinine continued to improve and within five days it returned to baseline form $13 \mathrm{mg} / \mathrm{dl}$ to $0.87 \mathrm{mg} / \mathrm{dl}$. On day five, repeat CT abdomen and pelvis showed only tiny punctate $(<3 \mathrm{~mm})$ non-obstructing calcification in the upper pole of right kidney. His nephrostomy tubes and Foley catheter were discontinued. Two day later, he had Lasix renogram which showed normal perfusion, concentration, and excretion by both kidneys with a split function of $44 \%$ left and $56 \%$ right. It did not show any obstruction to the flow of urine on either side. The patient was discharge home on day 10 (Table 1).

Table I Patient observations

\begin{tabular}{llllll}
\hline Days & Potassium $(\mathbf{m m o l} / \mathbf{L})$ & $\mathrm{CO}_{2}(\mathbf{m m o l} / \mathrm{L})$ & $\mathrm{BUN}(\mathrm{mg} / \mathrm{dL})$ & $\mathrm{Cr}(\mathrm{mg} / \mathrm{dl})$ & Phosphorus $(\mathbf{m g} / \mathbf{d l})$ \\
\hline Admission & 6 & 9 & 98 & 9.8 & 9.9 \\
Day \#2 & 4.1 & 20 & 51 & 3.17 & 6.1 \\
Day \#5 & 3.3 & 22 & 13 & 0.87 & 4.3 \\
Discharge & 3.9 & 23 & 15 & 0.85 & 3.5 \\
\hline
\end{tabular}

\section{Metabolism and pathophysiology}

Uric acid is a metabolic end product of purine compounds which can be found in excess either in purine-rich foods or rapid cell turnover in conditions such as tumor lysis syndrome. The major enzyme involved in this mechanism is xanthine oxidase which can be inhibited by purine analogue allopurinol or non-purine analogue febuxostat. One third of daily generated uric acid is metabolized by the gastrointestinal tract bacteria into carbon dioxide and ammonia. Rest of the uric acid is excreted by proximal tubules of kidneys. ${ }^{10}$ About $95 \%$ of serum uric acid is in monosodium urate form that is freely filtered in the glomeruli, out of which $99 \%$ gets reabsorbed in the proximal convoluted tubules. The $80 \%$ post secretory absorption occurs in the distal convoluted tubules that eventually results in only $10 \%$ excretion of the filtered urate in the urine. ${ }^{11}$

The humans lack Uricase enzyme (found in most mammals) that converts uric acid to allantoin which is $10-100$ times more soluble. ${ }^{12}$ At physiological $\mathrm{pH}$ of 7.40, uric acid mostly circulates in the ionized form as urate which is more soluble. At urine $\mathrm{pH}<5.5$, most of the uric acid becomes protonated which is poorly soluble and predisposes to uric acid nephrolithiasis. Two major mechanisms for acidic urinary $\mathrm{pH}$ are increased acid production or impaired renal ammoniagenesis. ${ }^{13}$

Type 2 diabetes results in markedly higher net acid excretion that leads to low urinary $\mathrm{pH}$ and has been associated with increased risk of uric acid stones. Obesity contributes more than dietary factor in nephrolithiasis. Since body fat is hydrophobic, in obesity, proportion of body water decreases and leads to dehydration. Furthermore, obesity is a pro-inflammatory state associated with electrolyte imbalance and altered urine chemistry. ${ }^{14}$ Many features of metabolic syndrome including hypertension, obesity, hyperglycemia, and dyslipidemia are associated with uric acid stone formers.

\section{Diagnosis}

Uric acid stones are more prevalent in older population. Detailed dietary and medical history such as gout, myeloproliferative disorder can suggest clues in uric acid nephrolithiasis diagnosis. The patients usually present with flank pain either dull aching or colicky along with nausea and vomiting, they may also be asymptomatic. When diagnosis is not clear or patient present with fevers, immediate imaging is recommended. An acidic urinary $\mathrm{pH}$, urine analysis, urine microscopy, 24-hours urine metabolic work up, and elevated serum uric acid levels can aid in the diagnosis. Besides uric acid stones, cysteine stones are the only other stones associated with acidic urine. The bedside Ultrasound sound can be used as first line diagnostic imaging tool due to its convenience, cost, and to eliminate radiation risk. However, it is only $45 \%$ sensitive and $88 \%$ specific for renal calculi. KUB is $44-47 \%$ sensitive and $80-87 \%$ specific. ${ }^{15}$

Non-contrast computerized tomography (CT) is the best modality in nephrolithiasis evaluation. It is $96.6 \%$ sensitive and $94.9 \%$ specific in diagnosing nephrolithiasis. ${ }^{15}$ On CT scan, uric acid stones appear dense and it can also help differentiating uric acid stones from papillary necrosis, transitional cell carcinoma, and fungal bezoars because all these appear radiolucent on routine radiographs, such as KUB. Other radiolucent stones include matrix, xanthine, hypoxanthine, 2,8-dihydroxyadenine, and indinavir stones. Indinavir is the only radiolucent stone that is not visualized on non-contrast $\mathrm{CT}$ scan. ${ }^{16}$ 


\section{Prevention and treatment}

Uric acid stones can be prevented with high fluid intake, low animal protein intake, urine alkalization to urine $\mathrm{pH}$ of 6.2-6.8, and xanthine oxidase inhibitors. Uric acid stones are among few urinary tract stones that can be dissolved successfully. The Treatment of uric acid nephrolithiasis consists of hydration to produce at least $2 \mathrm{~L}$ of urine volume daily, low animal protein diet, and alkalization of urine.

\section{Diet and fluid intake}

It is recommended to decrease daily animal protein intake to $0.8 \mathrm{~g} /$ $\mathrm{kg} /$ day and substitute with fresh fruits and vegetables to decrease uric acid production and provide urine alkalization, respectively. ${ }^{17}$ The high purines rich diet that should be avoided include meat, animal organs, yeast, sweetbreads, and fish.

Even though there are no interventional studies to observe effect of urine volume on uric acid stones, intuitively, it makes sense to increase fluid intake to produce at least $2.5 \mathrm{~L}$ urine which will reduce uric acid super-saturation. Studies have shown $40 \%-50 \%$ reduction in calcium stone recurrence with increased urinary volume. ${ }^{18}$

\section{Urinary alkalization via systemic means}

Oral alkalization for uric acid stone dissolution is effective in $80 \%$ of the patients. Sodium bicarbonate $650 \mathrm{mg}$ three times per day or commercial baking soda as an alternative with a dose of 1 to 2 teaspoons three times per day is inexpensive mode of alkalization. Even though both tolerated well, sodium alkali increases sodium and water load which is detrimental in patients with hypertension, congestive heart failure, and liver cirrhosis. Sodium load also increases sodium and calcium excretion in urine leading to calcium oxalate stones. ${ }^{19}$

Therefore, potassium citrate with a dose of $30-60$ meq per day is now considered as first line therapy in oral alkalization to treat and prevent uric acid stones.

\section{Local urinary alkalization (Chemolysis)}

Although majority of uric acid stones can be dissolved with systemic alkalization, there are situations when this cannot be accomplished such as, bilateral complete ureteral obstruction, severe metabolic disorders, severe renal failure, or fluid overload. In these cases, local dissolution of stones is an effective non-surgical approach. The percutaneous chemolysis to dissolve large uric acid stones was a common practice in the past. Due to prolonged hospitalization these procedures are not considered cost-effective. However, these procedures still have important non-surgical role in above mentioned situations.

It can be achieved either by retrograde or percutaneous irrigation. Earlier series of percutaneous chemolysis of renal calculi was reported by Pfister et al. ${ }^{20}$ Struvite, apatite, and carbonate stones can be dissolved with an acidic solution (hemiacidrin, Suby solution G) due to their $\mathrm{pH}$ of 4 . Cystine and uric acid stones can be dissolved with THAM-E (tromethamine, $\mathrm{pH}$ 10.2), Mucomyst (acetylcysteine, $\mathrm{pH} 8.2$ ), or sodium bicarbonate ( $\mathrm{pH} 8.2) .20$ In 1985, Sadi et al. ${ }^{21}$ devised an in vitro model to evaluate the efficacy of the different irrigating solutions utilized for local dissolution of uric acid stones. Tris aminomethane (THAM-E) was noted to be superior to sodium bicarbonate. They recommended the use of $0.3 \mathrm{M}$ concentration of THAM-E at flow rate of $50 \mathrm{cc} /$ hour as first line solution whenever local dissolution of uric acid stone is attempted. ${ }^{21}$
After 1990, there was recent case report from Serbia with percutaneous dissolution of uric acid stone in a patient with a solitary kidney. In the United States these procedures are not consider costeffective. However, physicians should be aware of this alternative especially in the situation as mentioned in our case report. Kachrilas et al..$^{22}$ also believe in role of percutaneous chemolysis. They presented review of 29 patients where $55 \%$ of the patients were stone free and $28 \%$ had partial dissolution with half of them showing nonsignificant $(<4 \mathrm{~mm})$ stone fragments after percutaneous chemolysis. ${ }^{22}$

\section{Carbonic anhydrase inhibitor}

A combination of sodium bicarbonate and a carbonic anhydrase inhibitor (Acetazolamide) has been used to improve urinary alkalization by inhibiting bicarbonate reabsorption in proximal tubules. However, acetazolamide may reduce urinary citrate and increase urinary phosphate that can lead to calcium phosphate stones. ${ }^{19}$

\section{Xanthine oxidase inhibitors}

Since hyperuricemia can cause hyperuricosuria, purine analogue allopurinol or non-purine analogue febuxostat can be used to inhibit xanthine oxidase. Xanthine oxidase converts hypoxanthine to xanthine and xanthine to uric acid. Hypoxanthine and xanthine are soluble and are excreted by kidneys. ${ }^{23}$

\section{Surgical management}

All lithotripsy modalities are effective for uric acid stone fragmentation. This may improve oral chemolysis by increasing the exposed stone surface. Open surgical extraction is the last treatment option for the nephrolithiasis.

\section{Conclusion}

The incidence of Uric acids stones can range from $4 \%$ in the United States to $40 \%$ in Israel due to its formation based on age, climate, ethnicity, and geographic location. These variations indicate that genes, diet, and environmental factors play major role in formation of uric acid stones. Acidic $\mathrm{pH}(<5.5)$ is a major risk factor in uric acid stone formation. Within stone formers, patients with history of obesity, diabetes, and metabolic syndrome are more prone to make uric acid stones. The Treatment of uric acid nephrolithiasis consists of hydration to increase urine volume, low animal protein diet, and urine alkalization. Although localized alkalization via retrograde or percutaneous is now an uncommon practice due to longer hospital stays and cost effectiveness. However, physicians should be aware of this effective alternative especially in situations with complete bilateral ureteral obstruction, severe metabolic disorders, acute renal failure, or fluid overload.

\section{Acknowledgements}

None.

\section{Conflict of interest}

Authors declare there is no conflict of interest in publishing the article.

\section{References}

1. Mandel NS, Mandel GS. Urinary tract stones disease in the United States veteran population. II. Geographic analysis of variations in composition. J Urol. 1989;142:1516-1521. 
2. Ma Q, Fang L, Rui S, et al. Uric acid stones, clinical manifestations and therapeutic considerations. PostGrad Med J. 2018;94(1114):458-462.

3. Gentle DL, Stoller ML, et al. Geriatric urolithiasis. $J$ Urol. 1997;158:2221-2224.

4. Borghi L, Meschi T, et al. Hot occupation and nephrolithiasis. J Urol. 1993;150(6):1757-1760.

5. Riese RJ, Sakhaee K. Uric acid nephrolithiasis: pathogenesis and treatment. J Urol. 1992;148:765-771.

6. Halabe A, Sperling O. Uric acid nephrolithiasis. Miner Electrolyte Metab. 1994;20(6):424-431.

7. Shekarriz B, Stoller ML. Uric acid nephrolithiasis: current concepts and controversies. J Urol. 2002;168(4):1307-1314.

8. Maalouf N, Cameron MA, et al. Novel insights into the pathogenesis of uric acid nephrolithiasis. Curr Opin Nephrol Hypertens. 2004:13(2):181-189.

9. Daudon M, Lacour B et al. High prevalence of uric acid calculi in diabetic stone formers. Nephrol Dial Transplant. 2005;20(2):468-469.

10. Mehta TH, Goldfarb D. Uric acid stones and hyperuricosuria. $A d v$ Chronic Kidney Dis. 2012;19(6):413-418.

11. Abou Elela A. Epidemiology, pathophysiology, and management of uric acid urolithiasis: A narrative reviw. J Adv Res. 2017;8(5):513-527.

12. Daudon M, Frochot V. Crystalluria. Clin Chem Lab Med. 2015:53(suppl 2):1479-1487.

13. Maalouf NM, Cameron MA, Moe OW, et al. Novel insights into the pathogenesis of uric acid nephrolithiasis. Curr Opin Nephrol Hypertens. 2004; $13: 181-189$
14. Livingston EH, Kohlstadt I. Simplified resting metabolic ratepredicting formulas for normal-sized and obese individuals. Obes Res. 2005; 13(7):1255-1262

15. Jel, Lison FC, Smith JC, et al. Effect of low dose radiation computerized tomography protocols on distal ureteral calculus detection. J Urol. 2009:182(6):2762-2767.

16. Resnick M I, Kursh E, et al. Use of computerized tomography in the delineation of uric acid calculi. J Urol. 1984;131(1):9-10.

17. MeschiT, Maggiore U,FiaccadoriE, etal. The effect of fruits and vegetables on urinary stone risk factors. Kidney Int. 2004;66(6):2402-2410.

18. Borghi L, Meschi T, et al. Urinary volume, water and recurrences in idiopathic calcium nephrolithiasis: a 5-year randomized prospective study. J Urol. 1996;155:839-843.

19. Freed SZ. The alternating use of an alkalizing salt and acetazolamide in the management of cysteine and uric acid stones. J Urol. 1975;113(1):96-99.

20. Pfister RC, Dretler SP. Percutaneous chemolysis of renal calculi. Urol Radiol. 1984;6(2):138-143.

21. Sadi M V, Saltzman N, Feria G, et al. Experimental observations on dissolution of uric acid calculi. J Urol. 1985;134:575-579.

22. Kachrilas S, Papatsoris A, et al. The current role of percutaneous chemolysis in the management of urolithiasis: review and results. Urolithiais. 2013;41(4):323-326.

23. Freeene, ML, Fujimoto WY, Seegmiller JE. Urinary xanthine stones- a rare complication of allopurinol therapy. $N$ Engl $\mathrm{J} \mathrm{Med}$. $1969 ; 280(8): 426-427$. 